\title{
Spatial behavior for solutions in heat conduction with two delays
}

\author{
M.C. Leseduarte and R. Quintanilla
}

Matemàtica Aplicada 2, ETSEIAT, Universitat Politècnica de Catalunya

Colom, 11. Terrassa (08222). Barcelona. Spain

E-mail addresses: Mari.Carme.Leseduarte@upc.edu, Ramon.Quintanilla@upc.edu

\begin{abstract}
In this note we investigate the spatial behavior of the solutions of the equation proposed to describe a theory for the heat conduction with two delay terms. We obtain an alternative of the Phragmén-Lindelöf type, which means that the solutions either decay or blow-up at infinity, both options in an exponential way. We also describe how to obtain an upper bound for the amplitude term. This is the first contribution on spatial behavior for partial differential equations involving two delay terms. We use energy arguments. The main point of the contribution is the use of an exponentially weighted energy function.
\end{abstract}

keywords: three dual-phase-lag heat conduction; two-temperatures heat conduction; spatial estimate; energy argument; equation with delay

\section{Introduction}

Spatial decay/growth of solutions for several thermomechanical situations has deserved much attention in the last decades. Decay estimates for elliptic [1], parabolic [2,3], hyperbolic [4-6] equations and/or combination of them $[7,8]$ have been obtained in this period. However, as far as the authors know, there are only two contributions $[9,10]$ to the case of partial differential equations with a delay term. In this paper we provide a new contribution to study the case of a partial differential equation with two delay terms.

Our result is relevant not only because we obtain an abstract contribution, but also because the equation we study has been considered as a possible model for the description of the heat conduction. There are several parabolic and hyperbolic theories that describe the heat conduction, where the propagation of heat is modeled as having finite speed, in contrast to the classical model using Fourier's law and leading to infinite propagation speed of heat signals. The articles by Chandrasekharaiah [11] or Hetnarski and Ignaczak [12,13] and the books by Ignaczak and Ostoja-Starzewski [14] or Straughan [15] are good reviews of the situation.

Recently, Tzou [16] proposed the dual-phase-lag heat equation. This theory is based on the constitutive equation

$$
\mathbf{q}\left(\mathbf{x}, t+\tau_{A}\right)=-k \nabla \theta\left(\mathbf{x}, t+\tau_{B}\right) \quad k>0 .
$$


Here, $\mathbf{q}$ is the heat flux vector and $\theta$ is the temperature. This equation shows that the temperature gradient established across a material volume at the position $\mathbf{x}$ at time $t+\tau_{B}$ results in a heat flux to flow at a different instant of time $t+\tau_{A}$. This delay is understood in terms of the microstructure of the material. Roy Choudhuri [17] extended this constitutive equation to

$$
\mathbf{q}\left(\mathbf{x}, t+\tau_{A}\right)=-\left(k \nabla \theta\left(\mathbf{x}, t+\tau_{B}\right)+k^{*} \nabla \nu\left(\mathbf{x}, t+\tau_{C}\right)\right), \quad k, k^{*}>0 .
$$

Here, $\nu$ is the thermal displacement that satisfies $\dot{\nu}=\theta$, and $\tau_{A}>\max \left\{\tau_{B}, \tau_{C}\right\}$. If we adjoin these constitutive equations with the energy equation

$$
-\nabla \mathbf{q}(\mathbf{x}, t)=\dot{\theta}(\mathbf{x}, t)
$$

we obtain the evolution equation

$$
\theta_{t t}(t)=k \Delta \theta_{t}\left(t-\tau_{1}\right)+k^{*} \Delta \theta\left(t-\tau_{2}\right)
$$

where $\tau_{1}:=\tau_{A}-\tau_{B}>0$ and $\tau_{2}:=\tau_{A}-\tau_{C}>0$. To simplify the expressions, we have taken the specific heat equal to one. In the work of Dreher et al. [18] (see also [19]) the authors proved that for this equation there always exists a sequence of eigenvalues in the point spectrum such that its real part tends to infinity. This result not only shows the instability of the problem, but what is worse, it also says that the associated mathematical problem with standard initial and boundary conditions is ill posed. In fact, the solutions could cease to exist after a finite time. The mathematical consequences of such theories do not agree with what one would expect $a$ priori. Thus, one thinks that this proposition is a very explosive candidate to describe the heat conduction.

If we want to set down a well posed problem with the constitutive equation, we can combine it with the theory proposed in the decade of 1960's through the contributions by Chen and Gurtin [20], Chen et al. [21,22] and Warren and Chen [23] as it was proposed and proved in the paper by Quintanilla [24]. Our paper follows this contribution. It is worth noting that several recent contributions have been addressed to the study of the qualitative aspects for the theory of Chen, Gurtin, Warren and Williams (see [25-28]). For the heat equation with one delay we may recall the contributions by Quintanilla [29] and Quintanilla and Jordan [30].

The main tool to obtain our results is the use of energy arguments. In particular, we believe that it is very relevant the use of a suitable weighted energy function $F_{\lambda, \omega}$, defined at the begining of Section 3 which satisfies the usual conditions to work with in the context of spatial estimates. The choice of this function has allowed to obtain a rate of decay for the solutions. In fact, we will prove that the solutions growth or decay as the exponential of $\pm z / \kappa$ does, where $\kappa$ is a positive function of time to be defined later (see (39)).

The plan of this note is the following. Section 2 is devoted to set down the problem we are going to study. A Phragmén-Lindelöf alternative for the solutions of the problem is obtained in Section 3. In Section 4, we obtain an upper bound for the amplitude term. Some conclusions end the manuscript.

\section{Basic equations}

In this section we propose the basic problem that we are going to study. We denote by $\theta$ the conductive temperature, $T$ is the thermodynamic temperature, $\nu$ is the conductive thermal displacement and $\alpha$ is the thermodynamic thermal displacement. 
The basic equations in this theory are (see [24]): the energy equation

$$
-\nabla \mathbf{q}(\mathbf{x}, t)=\dot{T}(\mathbf{x}, t)
$$

and the relations

$$
T=\theta-a \Delta \theta, \quad \alpha=\nu-a \Delta \nu,
$$

where $a$ is a positive constant. As we said in the introduction, we have taken the specific heat equal to one to simplify the expressions.

If we combine the equations, we obtain the field equation which governs the heat conduction in this theory

$$
\ddot{\nu}(t)-a \Delta \ddot{\nu}(t)=k \Delta \theta\left(t-\tau_{1}\right)+k^{*} \Delta \nu\left(t-\tau_{2}\right) .
$$

We here assume that $k, k^{*}, \tau_{1}$ and $\tau_{2}$ are positive parameters.

In this note we are going to work in a semi-infinite cylinder $B$ with a cross section $D$ that has a boundary smooth enough to guarantee the use of the divergence theorem.

We want to study the equation until a moment of time $t_{0}$. This assumption will be relevant, because our estimates depend on this instant.

The boundary conditions are:

$$
\begin{aligned}
& \nu(\mathbf{x}, t)=0, \quad(\mathbf{x}, t) \in(0, \infty) \times \partial D \times\left(0, t_{0}\right) \\
& \nu(\mathbf{x}, t)=\nu_{0}\left(x_{2}, x_{3}, t\right), \quad(\mathbf{x}, t) \in\{0\} \times D \times\left(0, t_{0}\right)
\end{aligned}
$$

where $\nu_{0}\left(x_{2}, x_{3}, t\right)$ is a given function. We also impose the null initial conditions:

$$
\nu(\mathbf{x}, s)=0, s \in\left(-\tau_{2}, 0\right], \mathbf{x} \in B \text { and } \theta(\mathbf{x}, s)=0, s \in\left(-\tau_{1}, 0\right], \mathbf{x} \in B .
$$

To guarantee the compatibility of the boundary and initial data, we shall assume that $\nu_{0}\left(x_{2}, x_{3}, 0\right)$ must vanish for every $\left(x_{2}, x_{3}\right) \in \partial D$.

In this work we analyze the problem determined by equation (2.3), boundary conditions (2.4), (2.5) and initial conditions (2.6). We will use the following fact (see the Appendix of [9] for a proof):

Exponentially weighted Poincaré inequality. Assume that $f(s)$ is a differentiable function in $[0, t]$ such that $f(0)=0$. Then, the following inequality

$$
\int_{0}^{t} \exp (-2 \omega s) f^{2}(s) d s \leq \frac{4 t^{2}}{\pi^{2}+4 t^{2} \omega^{2}} \int_{0}^{t} \exp (-2 \omega s)(\dot{f}(s))^{2} d s
$$

holds, for every $\omega>0$. We note that $\varphi(t)=\frac{4 t^{2}}{\pi^{2}+4 t^{2} \omega^{2}}$ is a growing function and so

$$
\int_{0}^{t} \exp (-2 \omega s) f^{2}(s) d s \leq \frac{4 t_{0}^{2}}{\pi^{2}+4 t_{0}^{2} \omega^{2}} \int_{0}^{t} \exp (-2 \omega s)(\dot{f}(s))^{2} d s, \text { for } 0<t \leq t_{0} .
$$




\section{$3 \quad$ Spatial estimates}

The aim of this section is to state a Phragmén-Lindelöf alternative for the solutions of the problem defined by (2.3)-(2.6). That is, we will prove that the solutions become unbounded in an exponential way or they decay as a negative exponential.

Let $\lambda$ and $\omega$ be two positive constants such that

$$
\omega>\frac{\lambda}{a^{2}}\left[\frac{k}{2}+k^{*} \frac{t_{0}}{\left(\pi^{2}+4 t_{0}^{2} \omega^{2}\right)^{1 / 2}}\right]^{2}+\frac{1}{a}\left[k+k^{*} \frac{2 t_{0}}{\left(\pi^{2}+4 t_{0}^{2} \omega^{2}\right)^{1 / 2}}\right] .
$$

We can guarantee the existence of the above parameters satisfying (3.1) because of Bolzano's theorem. We define the function

$$
F_{\lambda, \omega}(z, t)=-\int_{0}^{t} \int_{D} \exp (-2 \omega s)\left(a \ddot{\nu}_{, 1}(s)+k \dot{\nu}_{, 1}\left(s-\tau_{1}\right)+k^{*} \nu_{, 1}\left(s-\tau_{2}\right)\right)(\dot{\nu}(s)+\lambda \ddot{\nu}(s)) d A d s,
$$

for every $t \leq t_{0}$, where $z=x_{1}$. From the divergence theorem, we obtain that

$$
\begin{array}{r}
F_{\lambda, \omega}(z+h, t)=F_{\lambda, \omega}(z, t)-\frac{1}{2} \exp (-2 \omega t) \int_{z}^{z+h} \int_{D}\left[(\dot{\nu})^{2}+a(\nabla \dot{\nu})^{2}\right] d v- \\
-\int_{0}^{t} \int_{z}^{z+h} \int_{D} \exp (-2 \omega s)\left[\lambda(\ddot{\nu}(s))^{2}+a \lambda|\nabla \ddot{\nu}(s)|^{2}+\omega(\dot{\nu}(s))^{2}+\omega a|\nabla \dot{\nu}(s)|^{2}+\Phi\right] d v d s,
\end{array}
$$

for every $h>0$, where

$$
\Phi=k \nabla \dot{\nu}\left(s-\tau_{1}\right) \nabla \dot{\nu}(s)+\lambda k \nabla \dot{\nu}\left(s-\tau_{1}\right) \nabla \ddot{\nu}(s)+k^{*} \nabla \nu\left(s-\tau_{2}\right) \nabla \dot{\nu}(s)+\lambda k^{*} \nabla \nu\left(s-\tau_{2}\right) \nabla \ddot{\nu}(s) .
$$

If $F_{\lambda, \omega}(z, t) \rightarrow 0$ as $z \rightarrow \infty$, from (3.3) we have

$$
\begin{array}{r}
F_{\lambda, \omega}(z, t)=\frac{1}{2} \exp (-2 \omega t) \int_{B(z, \infty)}\left[(\dot{\nu})^{2}+a(\nabla \dot{\nu})^{2}\right] d v+ \\
+\int_{0}^{t} \int_{B(z, \infty)} \exp (-2 \omega s)\left[\lambda(\ddot{\nu}(s))^{2}+a \lambda|\nabla \ddot{\nu}(s)|^{2}+\omega(\dot{\nu}(s))^{2}+\omega a|\nabla \dot{\nu}(s)|^{2}+\Phi\right] d v d s,
\end{array}
$$

where $B(z, \infty)=\left\{\mathbf{x} \in B: x_{1}>z\right\}$.

From (3.3), we also see that the spatial derivative of $F_{\lambda, \omega}(z, t)$ is

$$
\begin{array}{r}
\frac{\partial F_{\lambda, \omega}(z, t)}{\partial z}=-\frac{1}{2} \exp (-2 \omega t) \int_{D}\left[(\dot{\nu})^{2}+a(\nabla \dot{\nu})^{2}\right] d A- \\
-\int_{0}^{t} \int_{D} \exp (-2 \omega s)\left[\lambda(\ddot{\nu}(s))^{2}+a \lambda|\nabla \ddot{\nu}(s)|^{2}+\omega(\dot{\nu}(s))^{2}+\omega a|\nabla \dot{\nu}(s)|^{2}+\Phi\right] d A d s .
\end{array}
$$

Taking into account the initial conditions (2.6) and the weighted Poincaré inequality (2.8), we 
get that

$$
\begin{array}{r}
\int_{0}^{t} \exp (-2 \omega s)\left[a \lambda|\nabla \ddot{\nu}(s)|^{2}+\omega a|\nabla \dot{\nu}(s)|^{2}+\Phi\right] d s \geq \\
a \lambda \int_{0}^{t} \exp (-2 \omega s)|\nabla \ddot{\nu}(s)|^{2} d s+ \\
+\left[\omega a-k-k^{*} \frac{2 t_{0}}{\left(\pi^{2}+4 t_{0}^{2} \omega^{2}\right)^{1 / 2}}\right] \int_{0}^{t} \exp (-2 \omega s)|\nabla \dot{\nu}(s)|^{2} d s- \\
-\lambda\left(k+k^{*} \frac{2 t_{0}}{\left(\pi^{2}+4 t_{0}^{2} \omega^{2}\right)^{1 / 2}}\right)\left(\int_{0}^{t} \exp (-2 \omega s)|\nabla \dot{\nu}(s)|^{2} d s\right)^{1 / 2} \times \\
\times\left(\int_{0}^{t} \exp (-2 \omega s)|\nabla \ddot{\nu}(s)|^{2} d s\right)^{1 / 2} .
\end{array}
$$

Thus,

$$
\begin{array}{r}
\int_{0}^{t} \exp (-2 \omega s)\left[a \lambda|\nabla \ddot{\nu}(s)|^{2}+\omega a|\nabla \dot{\nu}(s)|^{2}+\Phi\right] d s \geq \\
\geq \mu^{*}\left[\int_{0}^{t} \exp (-2 \omega s)|\nabla \dot{\nu}(s)|^{2} d s+\int_{0}^{t} \exp (-2 \omega s)|\nabla \ddot{\nu}(s)|^{2} d s\right],
\end{array}
$$

where

$$
\begin{array}{r}
\mu^{*}=\frac{1}{2}\left[a \lambda+\omega a-k-k^{*} \frac{2 t_{0}}{\left(\pi^{2}+4 t_{0}^{2} \omega^{2}\right)^{1 / 2}}\right] \\
-\frac{1}{2} \sqrt{\left[a \lambda-\omega a+k+k^{*} \frac{2 t_{0}}{\left(\pi^{2}+4 t_{0}^{2} \omega^{2}\right)^{1 / 2}}\right]^{2}+4 \lambda^{2}\left[\frac{k}{2}+k^{*} \frac{t_{0}}{\left(\pi^{2}+4 t_{0}^{2} \omega^{2}\right)^{1 / 2}}\right]^{2}} .
\end{array}
$$

We note that $\mu^{*}$ is the lowest eigenvalue of the symmetric matrix

$$
\left(\begin{array}{cc}
a \lambda & -\lambda\left(\frac{k}{2}+k^{*} \frac{t_{0}}{\left(\pi^{2}+4 t_{0}^{2} \omega^{2}\right)^{1 / 2}}\right) \\
-\lambda\left(\frac{k}{2}+k^{*} \frac{t_{0}}{\left(\pi^{2}+4 t_{0}^{2} \omega^{2}\right)^{1 / 2}}\right) & \omega a-k-k^{*} \frac{2 t_{0}}{\left(\pi^{2}+4 t_{0}^{2} \omega^{2}\right)^{1 / 2}}
\end{array}\right)
$$

which is positive because of the condition (3.1). From (3.6) and (3.8) we have

$$
\frac{\partial F_{\lambda, \omega}(z, t)}{\partial z} \leq-\mu^{*} \int_{0}^{t} \int_{D} \exp (-2 \omega s)\left[|\nabla \dot{\nu}(s)|^{2}+|\nabla \ddot{\nu}(s)|^{2}\right] d A d s .
$$

Now we will estimate the absolute value of $F_{\lambda, \omega}(z, t)$ in terms of its spatial derivative. We have that

$$
\left|F_{\lambda, \omega}(z, t)\right| \leq \sum_{i=1}^{6} I_{i}
$$


where

$$
\begin{aligned}
& I_{1}=a \int_{0}^{t} \int_{D} \exp (-2 \omega s)\left|\ddot{\nu}_{, 1}(s)\right||\dot{\nu}(s)| d A d s, \\
& I_{2}=a \lambda \int_{0}^{t} \int_{D} \exp (-2 \omega s)\left|\ddot{\nu}_{, 1}(s)\right||\ddot{\nu}(s)| d A d s, \\
& I_{3}=k \int_{0}^{t} \int_{D} \exp (-2 \omega s)\left|\dot{\nu}_{, 1}\left(s-\tau_{1}\right)\right||\dot{\nu}(s)| d A d s, \\
& I_{4}=\lambda k \int_{0}^{t} \int_{D} \exp (-2 \omega s)\left|\dot{\nu}_{, 1}\left(s-\tau_{1}\right)\right||\ddot{\nu}(s)| d A d s, \\
& I_{5}=k^{*} \int_{0}^{t} \int_{D} \exp (-2 \omega s)\left|\nu_{, 1}\left(s-\tau_{2}\right)\right||\dot{\nu}(s)| d A d s, \\
& I_{6}=k^{*} \lambda \int_{0}^{t} \int_{D} \exp (-2 \omega s)\left|\nu_{, 1}\left(s-\tau_{2}\right)\right||\ddot{\nu}(s)| d A d s .
\end{aligned}
$$

We also have

$$
\begin{aligned}
I_{1} & \leq \frac{1}{2} \alpha_{1} a \int_{0}^{t} \int_{D} \exp (-2 \omega s)\left[|\nabla \dot{\nu}(s)|^{2}+|\nabla \ddot{\nu}(s)|^{2}\right] d A d s \\
I_{2} & \leq \alpha_{1} a \lambda \int_{0}^{t} \int_{D} \exp (-2 \omega s)|\nabla \ddot{\nu}(s)|^{2} d A d s \\
I_{3} & \leq \alpha_{1} k \int_{0}^{t} \int_{D} \exp (-2 \omega s)|\nabla \dot{\nu}(s)|^{2} d A d s, \\
I_{4} & \leq \frac{1}{2} \alpha_{1} k \lambda \int_{0}^{t} \int_{D} \exp (-2 \omega s)\left[|\nabla \dot{\nu}(s)|^{2}+|\nabla \ddot{\nu}(s)|^{2}\right] d A d s \\
I_{5} & \leq \alpha_{1} k^{*} \frac{2 t_{0}}{\left(\pi^{2}+4 t_{0}^{2} \omega^{2}\right)^{1 / 2}} \int_{0}^{t} \int_{D} \exp (-2 \omega s)|\nabla \dot{\nu}(s)|^{2} d A d s, \\
I_{6} & \leq \alpha_{1} k^{*} \lambda \frac{t_{0}}{\left(\pi^{2}+4 t_{0}^{2} \omega^{2}\right)^{1 / 2}} \int_{0}^{t} \int_{D} \exp (-2 \omega s)\left[|\nabla \dot{\nu}(s)|^{2}+|\nabla \ddot{\nu}(s)|^{2}\right] d A d s .
\end{aligned}
$$

Here, $\alpha_{1}^{2}$ is the inverse of the lowest eigenvalue of the problem

$$
\Delta u+\beta u=0 \text { on } D, \quad u=0 \text { on } \partial D .
$$

Then, it follows that

$$
\left|F_{\lambda, \omega}(z, t)\right| \leq-\kappa \frac{\partial F_{\lambda, \omega}(z, t)}{\partial z}
$$

where

$$
\kappa=\frac{\alpha_{1}(\lambda+1)}{\mu^{*}}\left(a+k+k^{*} \frac{2 t_{0}}{\left(\pi^{2}+4 t_{0}^{2} \omega^{2}\right)^{1 / 2}}\right) .
$$

We point out that we could improve the value for $\kappa$. However, to make the calculations easier, we prefer to set down the analysis in a clear way. It is worth noting that the use of the Poincaré inequality (on the cross section) is not strictly needed and then, the analysis could be also applied in the one dimensional case. 
Inequality (3.26) is well known in the study of spatial estimates. We have these two inequalities (see [1])

$$
\begin{gathered}
-\frac{\partial F_{\lambda, \omega}(z, t)}{\partial z} \geq \kappa^{-1}\left(-F_{\lambda, \omega}(z, t)\right), \\
-\frac{\partial F_{\lambda, \omega}(z, t)}{\partial z} \geq \kappa^{-1} F_{\lambda, \omega}(z, t) .
\end{gathered}
$$

If there is some $z_{0} \geq 0$ such that $F_{\lambda, \omega}\left(z_{0}, t\right)<0$, then $F_{\lambda, \omega}(z, t)<0$ for $z \geq z_{0}$. From (3.28) we obtain

$$
-F_{\lambda, \omega}(z, t) \geq-F_{\lambda, \omega}\left(z_{0}, t\right) \exp \left(\frac{z-z_{0}}{\kappa}\right), \quad z \geq z_{0} .
$$

Thus, when $F_{\lambda, \omega}\left(z_{0}, t\right)<0$, we conclude that

$$
\lim _{z \rightarrow \infty} \exp \left(-\frac{z}{\kappa}\right) H(z, t) \geq Q>0
$$

where

$$
\begin{array}{r}
H(z, t)=\frac{1}{2} \exp (-2 \omega t) \int_{B(z)}\left[(\dot{\nu})^{2}+a(\nabla \dot{\nu})^{2}\right] d v+ \\
+\int_{0}^{t} \int_{B(z)} \exp (-2 \omega s)\left[\lambda(\ddot{\nu}(s))^{2}+a \lambda|\nabla \ddot{\nu}(s)|^{2}+\omega(\dot{\nu}(s))^{2}+\omega a|\nabla \dot{\nu}(s)|^{2}+\Phi\right] d v d s,
\end{array}
$$

and $B(z)=\left\{\mathbf{x} \in B: 0 \leq x_{1} \leq z\right\}$.

Otherwise, we have that $F_{\lambda, \omega}(z, t) \geq 0$ for every $z \geq 0$. A quadrature on (3.29) yields

$$
F_{\lambda, \omega}(z, t) \leq F_{\lambda, \omega}(0, t) \exp \left(-\frac{z}{\kappa}\right), \quad z \geq 0 .
$$

We can summarize the results in the following way:

Theorem 3.1 Let $\nu(\mathbf{x}, t)$ be a solution of the problem defined by (2.3)-(2.6). Then either the function $\nu(\mathbf{x}, t)$ satisfies the asymptotic condition (3.31) or it satisfies the decay estimate (3.33).

We note that estimate (3.33) and (3.5) imply that the estimate

$$
\begin{array}{r}
\frac{1}{2} \int_{B(z, \infty)}\left[(\dot{\nu})^{2}+a(\nabla \dot{\nu})^{2}\right] d v+ \\
+\int_{0}^{t} \int_{B(z, \infty)}\left[\lambda(\ddot{\nu}(s))^{2}+a \lambda|\nabla \ddot{\nu}(s)|^{2}+\omega(\dot{\nu}(s))^{2}+\omega a|\nabla \dot{\nu}(s)|^{2}+\Phi\right] d v d s \leq \\
\leq F_{\lambda, \omega}(0, t) \exp \left(2 \omega t-\frac{z}{\kappa}\right)
\end{array}
$$

holds, for every $z \geq 0$.

Remark. To obtain our estimates, we have used that $\omega$ satisfies condition (3.1) in a relevant way. When the parameter $a$ tends to zero, the parameter $\omega$ should be unbounded and then, our arguments do not work. Consequently, we cannot use the analysis when $a=0$, which corresponds to the heat equation proposed by Roy Choudhuri. 


\section{The amplitude term}

The aim of this section is to describe how the amplitude term can be bounded in terms of the boundary data. We will show how to obtain an estimate, but we do not pay much attention to calculate the optimal values of the different parameters.

Let $\eta(\mathbf{x}, t)$ be a smooth function which satisfies the boundary conditions (2.4)-(2.5) and decays to zero in a uniform way when $x_{1}$ tends to infinity. We note that

$$
\begin{array}{r}
F_{\lambda, \omega}(0, t)= \\
-\int_{0}^{t} \int_{D} \exp (-2 \omega s)\left(a \ddot{\nu}_{, 1}(s)+k \dot{\nu}_{, 1}\left(s-\tau_{1}\right)+k^{*} \nu_{, 1}\left(s-\tau_{2}\right)\right)(\dot{\eta}(s)+\lambda \ddot{\eta}(s)) d A d s
\end{array}
$$

The divergence theorem gives

$$
\begin{array}{r}
F_{\lambda, \omega}(0, t)=\int_{0}^{t} \int_{B} \exp (-2 \omega s) \ddot{\nu}(s)(\dot{\eta}(s)+\lambda \ddot{\eta}(s)) d v d s \\
+\int_{0}^{t} \int_{B} \exp (-2 \omega s)\left(a \ddot{\nu}_{, i}(s)+k \dot{\nu}_{, i}\left(s-\tau_{1}\right)+k^{*} \nu_{, i}\left(s-\tau_{2}\right)\right)\left(\dot{\eta}_{, i}(s)+\lambda \ddot{\eta}_{, i}(s)\right) d v d s .
\end{array}
$$

We also have that

$$
\begin{array}{r}
\int_{0}^{t} \int_{B} \exp (-2 \omega s) \ddot{\nu}(s)(\dot{\eta}(s)+\lambda \ddot{\eta}(s)) d v d s \leq \varepsilon_{1} \int_{0}^{t} \int_{B} \exp (-2 \omega s)|\nabla \ddot{\nu}(s)|^{2} d v d s \\
+M_{1} \int_{0}^{t} \int_{B} \exp (-2 \omega s)(\dot{\eta}(s)+\lambda \ddot{\eta}(s))(\dot{\eta}(s)+\lambda \ddot{\eta}(s)) d v d s
\end{array}
$$

and

$$
\begin{array}{r}
\int_{0}^{t} \int_{B} \exp (-2 \omega s)\left(a \ddot{\nu}_{, i}(s)+k \dot{\nu}_{, i}\left(s-\tau_{1}\right)+k^{*} \nu_{, i}\left(s-\tau_{2}\right)\right)\left(\dot{\eta}_{, i}(s)+\lambda \ddot{\eta}_{, i}(s)\right) d v d s \leq \\
\leq \varepsilon_{2} \int_{0}^{t} \int_{B} \exp (-2 \omega s)\left(a \ddot{\nu}_{, i}(s)+k \dot{\nu}_{, i}\left(s-\tau_{1}\right)+k^{*} \nu_{, i}\left(s-\tau_{2}\right)\right) \\
\left(a \ddot{\nu}_{, i}(s)+k \dot{\nu}_{, i}\left(s-\tau_{1}\right)+k^{*} \nu_{, i}\left(s-\tau_{2}\right)\right) d v d s+ \\
+M_{2} \int_{0}^{t} \int_{B} \exp (-2 \omega s)\left(\dot{\eta}_{, i}(s)+\lambda \ddot{\eta}_{, i}(s)\right)\left(\dot{\eta}_{, i}(s)+\lambda \ddot{\eta}_{, i}(s)\right) d v d s \leq \\
\leq 3 \varepsilon_{2} \int_{0}^{t} \int_{B} \exp (-2 \omega s)\left[a^{2}|\nabla \ddot{\nu}(s)|^{2}+\left(k^{2}+\left(k^{*}\right)^{2} \frac{4 t_{0}^{2}}{\pi^{2}+4 t_{0}^{2} \omega^{2}}\right)|\nabla \dot{\nu}(s)|^{2}\right] d v d s+ \\
+M_{2} \int_{0}^{t} \int_{B} \exp (-2 \omega s)\left(\dot{\eta}_{, i}(s)+\lambda \ddot{\eta}_{, i}(s)\right)\left(\dot{\eta}_{, i}(s)+\lambda \ddot{\eta}_{, i}(s)\right) d v d s .
\end{array}
$$

Here, $\varepsilon_{i}, i=1,2$ are two positive constants which can be chosen as small as we want and $M_{i}$, $i=1,2$ are computable constants that depend on $\varepsilon_{i}$ and the geometry of the cross-section. If we select $\varepsilon_{i}, i=1,2$ small enough to guarantee that

$$
\varepsilon_{1}+3 \varepsilon_{2}\left[a^{2}+k^{2}+\left(k^{*}\right)^{2} \frac{4 t_{0}^{2}}{\pi^{2}+4 t_{0}^{2} \omega^{2}}\right]<\frac{\mu^{*}}{2},
$$


we obtain that

$$
\begin{aligned}
F_{\lambda, \omega}(0, t) & \leq 2 M_{1} \int_{0}^{t} \int_{B} \exp (-2 \omega s)(\dot{\eta}(s)+\lambda \ddot{\eta}(s))(\dot{\eta}(s)+\lambda \ddot{\eta}(s)) d v d s+ \\
+ & 2 M_{2} \int_{0}^{t} \int_{B} \exp (-2 \omega s)\left(\dot{\eta}_{, i}(s)+\lambda \ddot{\eta}_{, i}(s)\right)\left(\dot{\eta}_{, i}(s)+\lambda \ddot{\eta}_{, i}(s)\right) d v d s
\end{aligned}
$$

If we select

$$
\eta(\mathbf{x}, t)=\exp \left(-\xi x_{1}\right) \nu_{0}\left(x_{2}, x_{3}, t\right),
$$

where $\xi$ in an arbitrary positive constant, we compute

$$
\begin{aligned}
\dot{\eta}(\mathbf{x}, s) & =\exp \left(-\xi x_{1}\right) \dot{\nu}_{0}\left(x_{2}, x_{3}, s\right) \\
\ddot{\eta}(\mathbf{x}, s) & =\exp \left(-\xi x_{1}\right) \ddot{\nu}_{0}\left(x_{2}, x_{3}, s\right) \\
\dot{\eta}_{, 1}(\mathbf{x}, s) & =-\xi \exp \left(-\xi x_{1}\right) \dot{\nu}_{0}\left(x_{2}, x_{3}, s\right) \\
\dot{\eta}_{, \gamma}(\mathbf{x}, s) & =\exp \left(-\xi x_{1}\right) \dot{\nu}_{0, \gamma}\left(x_{2}, x_{3}, s\right), \gamma=2,3
\end{aligned}
$$

and

$$
\begin{aligned}
& \ddot{\eta}_{, 1}(\mathbf{x}, s)=-\xi \exp \left(-\xi x_{1}\right) \ddot{\nu}_{0}\left(x_{2}, x_{3}, s\right), \\
& \ddot{\eta}_{, \gamma}(\mathbf{x}, s)=\exp \left(-\xi x_{1}\right) \ddot{\nu}_{0, \gamma}\left(x_{2}, x_{3}, s\right), \gamma=2,3 .
\end{aligned}
$$

From (4.6), we obtain that

$$
\begin{array}{r}
F_{\lambda, \omega}(0, t) \leq \frac{M_{1}+\xi^{2} M_{2}}{\xi} \int_{0}^{t} \int_{D} \exp (-2 \omega s)\left(\dot{\nu}_{0}+\lambda \ddot{\nu}_{0}\right)^{2} d A d s+ \\
+\frac{M_{2}}{\xi} \int_{0}^{t} \int_{D} \exp (-2 \omega s)\left(\dot{\nu}_{0, \gamma}+\lambda \ddot{\nu}_{0, \gamma}\right)\left(\dot{\nu}_{0, \gamma}+\lambda \ddot{\nu}_{0, \gamma}\right) d A d s
\end{array}
$$

where $\gamma=2,3$. We point out that the RHS of (4.10) depends on $\xi$. This arbitrary positive constant can be selected as we want to optimize the upper bound. However, we do not include here this calculation.

\section{Conclusions}

In this paper we have investigated the spatial behavior of solutions for a partial differential equation with two delays. This equation came from the study of the heat conduction. It is worth noting that this is the first contribution concerning spatial behavior for a partial differential equation with two delays. We have also obtained an upper bound for the amplitude term by means of the boundary data.

\section{Acknowledgments}

This work is part of the project "Análisis Matemático de las Ecuaciones en Derivadas Parciales de la Termomecánica" (MTM2013-42004-P) of the Spanish Ministry of Economy and Competitiveness. 


\section{References}

[1] Flavin, J.N., Knops, R.J., Payne, L.E., 1989. Decay estimates for the constrained elastic cylinder of variable cross-section. Q. Appl. Math. 47, 325-350.

[2] Horgan, C.O., Payne, L.E., Wheeler, L.T., 1984. Spatial decay estimates in transient heat conduction. Q. Appl. Math. 42, 119-127.

[3] Horgan, C.O., Quintanilla, R., 2001. Spatial decay of transient end effects in functionally graded heat conducting materials. Q. Appl. Math. 59, 529-542.

[4] Chirita, S., Quintanilla, R., 1996. Saint-Venant's principle in linear elastodynamics. J. Elast. 42, 201-215.

[5] Flavin, J.N., Knops, R.J., Payne, L.E., 1989. Energy bounds in dynamical problems for a semi-infinite elastic beam. In: Elasticity: Mathematical Methods and Applications (Eason, G., Ogden, R. W. eds.), Chichester: Ellis Horwood, pp. 101-111.

[6] Horgan, C.O., Quintanilla, R., 2005. Spatial behaviour of solutions of the dual-phase-lag heat equations, Math. Methods Appl. Sci. 28, 43-57.

[7] Quintanilla, R., 2001. Damping of end effects in a thermoelastic theory. Appl. Math. Letters. 14, 137-141.

[8] Chirita, S., Ciarletta, M., 1999. Time-weighted surface power function method for the study of spatial behaviour in dynamics of continua, Eur. J. Mech. A/Solids. 18, 915-933.

[9] Leseduarte, M.C., Quintanilla, R., 2013. Phragmén-Lindelöf alternative for an exact heat conduction equation with delay, Commun. Pure Appl. Anal. 12, 1221-1235.

[10] Quintanilla, R., 2010. Spatial estimates for an equation with a delay term. J. Appl. Math. Phys. (ZAMP). 61, 381-388.

[11] Chandrasekharaiah, D.S., 1998. Hyperbolic thermoelasticity: A review of recent literature, Appl. Mech. Rev. 51, 705-729.

[12] Hetnarski, R.B., Ignaczak, J., 1999. Generalized thermoelasticity, J. Therm. Stresses. 22, 451-470.

[13] Hetnarski, R.B., Ignaczak, J., 2000. Nonclassical dynamical thermoelasticity, Int. J. of Solids and Structures. 37, 215-224.

[14] Ignaczak, J., Ostoja-Starzewski, M., 2010. Thermoelasticity with Finite Wave Sppeds, Mathematical Monographs. Oxford.

[15] Straughan, B., 2011. Heat Waves, Applied Mathematical Sciences, 177. Springer, New York.

[16] Tzou, D.Y., 1995. A unified approach for heat conduction from macro to micro-scales, ASME J. Heat Transf. 117, 8-16. 
[17] Roy Choudhuri, S.K., 2007. On a thermoelastic three-phase-lag model. J. Therm. Stresses. $30,231-238$.

[18] Dreher, M., Quintanilla, R., Racke, R., 2009. Ill posed problems in thermomechanics, Appl. Math. Letters. 22, 1374-1379.

[19] Jordan, P.M., Dai, W., Mickens, R.E., 2008. A note on the delayed heat equation: Instability with respect to initial data, Mech. Res. Commun. 35, 414-420.

[20] Chen, P.J., Gurtin, M.E., 1968. On a theory of heat involving two temperatures, J. Appl. Math. Phys. (ZAMP). 19, 614-627.

[21] Chen, P.J., Gurtin, M.E., Williams, W.O., 1968. A note on non-simple heat conduction, J. Appl. Math. Phys. (ZAMP). 19, 969-970.

[22] Chen, P.J., Gurtin, M.E., Williams, W.O., 1969. On the thermodynamics of non-simple materials with two temperatures, J. Appl. Math. Phys. (ZAMP). 20, 107-112.

[23] Warren, W.E., Chen, P.J., 1973. Wave propagation in two temperatures theory of thermoelaticity. Acta Mechanica. 16, 83-117.

[24] Quintanilla, R., 2009. A well-posed problem for the three-dual-phase-lag heat conduction. J. Therm. Stresses. 32, 1270-1278.

[25] Horgan, C.O., Quintanilla, R., 2005. Spatial decay of transient end effects for nonstandard linear diffusion problems, IMA J. Appl. Math. 70, 119-128.

[26] Horgan, C.O., Wheeler, L.T., 1975. A spatial decay estimate for pseudoparbolic equations, Letters in Appl. Eng. Sci. 3, 237-243.

[27] Quintanilla, R., 2004. Exponential stability and uniqueness in thermoelasticity with two temperatures. Dyn. Cont., Discrete and Impulsive Syst. A. 11, 57-68.

[28] Quintanilla, R., 2004. On existence, structural stability, convergence and spatial behaviour in thermoelasticity with two temperatures. Acta Mechanica. 68, 61-73.

[29] Quintanilla, R., 2011. Some solutions for a family of exact phase-lag heat conduction problems. Mech. Res. Commun. 38, 355-360.

[30] Quintanilla, R., Jordan, P.M., 2009. A note on the two temperature theory with dualphase-lag delay. Some exact solutions. Mech. Res. Commun. 36, 796-803. 